\title{
ARTICLE
}

\section{Measurement of photon dose rates in moderated neutron calibration fields}

\author{
Tadayoshi Yoshida $^{\mathrm{a}^{*}}$, Norio Tsujimura ${ }^{\mathrm{a}}$ and Hideaki Miyata ${ }^{\mathrm{b}}$ \\ ${ }^{a}$ Japan Atomic Energy Agency, 4-33 Muramatsu, Tokai-mura, Naka-gun, Ibaraki-ken, 319-1194, Japan; ${ }^{b}$ E\&E Techno \\ Service Co., Ltd., 4-33 Muramatsu, Tokai-mura, Naka-gun, Ibaraki-ken, 319-1194, Japan
}

The authors performed a complementary study to determine the proportion of the contaminating photons present in a moderated neutron field produced by a ${ }^{252} \mathrm{Cf}$ neutron source surrounded by moderators. The photon dose rates were measured with three types of instruments based on different detection principles, and the results were compared with those obtained from Monte Carlo calculation. The contaminant photon dose rates observed from each instrument were in good agreement, and the measurement and calculation results also agreed well.

Keywords: Contaminant photon; Monte Carlo; MCNP; moderated neutron calibration field; Geiger-Müller dosemeter; thermoluminescence dosemeter; ionization chamber dosemeter

\section{Introduction}

Neutron fields are always accompanied by gamma rays originating from both neutron-generating reactions and secondary neutron interactions in the surrounding media. For example, a spontaneous fission neutron source of ${ }^{252} \mathrm{Cf}$ emits prompt fission gamma rays and equilibrium fission product gamma rays with a gamma-to-neutron dose equivalent ratio of $\sim 5 \%$, which increases to $\sim 18 \%$ when the source is placed at the center of a 30-cm-diameter heavy-water sphere covered with a cadmium shell [1]. Most dosemeters respond to both neutrons and gamma rays to some degree, especially all gamma-ray dosemeters, which are rather responsive to neutrons; therefore, it is important to determine the separate neutron and gamma-ray components of the dose equivalents in any mixed neutron-gamma fields.

The Nuclear Fuel Cycle Engineering Laboratories (NCL) of the Japan Atomic Energy Agency (JAEA) built a moderated neutron calibration field using a ${ }^{252} \mathrm{Cf}$ neutron source and moderators made of acrylic resin, steel, and a combination of them [2,3]. The reference neutron dosimetry was already completed by both neutron spectrometry and spectral computation, but the photon dosimetry was not yet completed. We therefore embarked on the present complementary study to determine the proportion of contaminating photons present in the neutron calibration field by using three photon dosemeters with different detection principles and Monte Carlo photon spectral simulations. This paper summarizes the reference photon dosimetry, including a brief description of the NCL-moderated neutron field,

*Corresponding author. Email: yoshida.tadayoshi@jaea.go.jp method and results.

\section{Outline of moderated neutron calibration fields}

A moderated ${ }^{252} \mathrm{Cf}$ neutron field constructed at the Instrument Calibration Facility (ICF) of the NCL was characterized in terms of the gamma-ray dose components. Given below is a brief description of the facility, the source and moderator configurations.

The neutron irradiation room is $13.0 \times 12.5 \mathrm{~m}^{2}$ with a ceiling height of $5.3 \mathrm{~m}$. The outside-facing roof and walls are made of autoclaved aerated concrete with a thickness of $10 \mathrm{~cm}$ as a means for reducing room-scattered neutrons. The room has a $6 \times 6 \mathrm{~m}^{2}$ steel grating floor that acts as a pseudo ceiling for a basement room with an $8 \times 12 \mathrm{~m}^{2}$ concrete floor and four concrete walls of $2-\mathrm{m}$ in "depth". A ${ }^{252} \mathrm{Cf}$ source ( $\left.1 \mathrm{GBq}\right)$ of known neutron emission rate was remotely moved by a pneumatic source transfer system through a guide tube from a storage vault to an irradiation position at a height of $1.26 \mathrm{~m}$ from the grating floor. Annular cylinders made of steel and polymethyl methacrylate were placed around the source to alter the initial fission neutron spectrum. This configuration produced neutron fields that simulated the spectrum of neutrons transmitted through fuel-handling gloveboxes and their shielding windows. These spectra were a composite of the fission spectrum and the low-energy component caused by elastic scattering with hydrogen. The neutron spectrometry and dosimetry studies for each source configuration have been completed and described in greater detail elsewhere [2,3]. Table 1 lists the moderator configurations, along with their abbreviated 
identification codes, and the neutron field parameters $\bar{E}$ (fluence-averaged energy, $\mathrm{MeV}$ ) and $\bar{h}$ (spectrum-averaged ambient dose equivalent conversion

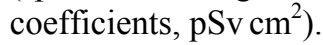

Table 1. Moderator configurations and neutron dosimetric parameters at a distance of $1 \mathrm{~m}$.

\begin{tabular}{|c|c|c|c|c|}
\hline \multirow{2}{*}{$\begin{array}{l}\text { Moderator } \\
\text { ID code }\end{array}$} & \multicolumn{2}{|c|}{ Moderator thickness $(\mathrm{mm})$} & \multirow{2}{*}{$\begin{array}{c}\bar{E} \\
(\mathrm{MeV})\end{array}$} & \multirow{2}{*}{$\begin{array}{c}\bar{h} \\
\left(\mathrm{pSv} \mathrm{cm}^{2}\right)\end{array}$} \\
\hline & Steel $^{(\mathrm{a})}$ & Acrylic $^{(b)}$ & & \\
\hline $\mathrm{Cf}$ & - & - & 2.0 & 380 \\
\hline P15 & - & 15 & 1.7 & 333 \\
\hline P35 & - & 35 & 1.4 & 272 \\
\hline P60 & - & 60 & 1.2 & 222 \\
\hline P100 & - & 100 & 0.96 & 178 \\
\hline F40 & 40 & - & 1.5 & 361 \\
\hline F40P60 & 40 & 60 & 0.85 & 187 \\
\hline
\end{tabular}

(a) Annular steel with an outer diameter of $180 \mathrm{~mm}$ and a height of $300 \mathrm{~mm}$.

(b) Annular acrylic resin with an outer diameter of $300 \mathrm{~mm}$ and a height of $300 \mathrm{~mm}$. F40P60 consists of $40 \mathrm{~mm}$ of steel nested into $60 \mathrm{~mm}$ of acrylic resin.

A $1 \mu$ g quantity of ${ }^{252} \mathrm{Cf}$ emits both $2.31 \times 10^{6}$ neutrons per second and $1.3 \times 10^{7}$ gamma rays per second. The latter is composed of approximately $50 \%$ prompt fission gamma rays and $50 \%$ equilibrium fission product gamma rays, depending to some degree on the details of the construction for encapsulation [4]. As mentioned in Introduction, these gamma rays when used bare deliver $\sim 5 \%$ of the dose equivalent rate of the neutron dose equivalent rate; however, the actual spectrum and intensity of these gamma rays are highly modified by self-absorption and interactions within the source transfer system and the moderator configurations.

\section{Gamma dosimetry methods}

\subsection{Measurements}

The photon dose rates were measured with three types of instruments based on different detection principles: a Geiger-Müller (GM) counter/dosemeter, a thermoluminescence dosemeter (TLD), and an ionization chamber. These instruments were selected because the neutron sensitivities of the former two are negligible and that of the latter one is compensatable (see Sections 3.1.1 to 3.1.3).

Each instrument was placed at a distance of $1 \mathrm{~m}$ from the center of the neutron source after calibration using a ${ }^{137} \mathrm{Cs}$ gamma-ray calibration field for the reason described later.

\subsubsection{GM detector}

The GM detector, model GM-1, is a commercial product manufactured by Far West Technology, Inc., USA, as a neutron-insensitive photon dosemeter used in a mixed photon-neutron field. It has been patterned after the work [5] of Wagner and Hurst, and detectors similar in construction have been used to measure the gamma rays mixed in neutron fields $[6,7,8]$. The GM tube is covered with $90 \mathrm{mg} \mathrm{cm}^{-2} \mathrm{Fe}, 807 \mathrm{mg} \mathrm{cm}^{-2} \mathrm{~Pb}$ and 200 $\mathrm{mg} \mathrm{cm}{ }^{-2} \mathrm{Al}$ for compensating the energy dependence and with $480 \mathrm{mg} \mathrm{cm}{ }^{-2}{ }^{6} \mathrm{LiF}$ for shielding thermal neutrons. The gamma energy response for air kerma is flat within $\pm 10 \%$ from $70 \mathrm{keV}$ to $\sim 2 \mathrm{MeV}$, and the neutron sensitivity is less than $1 \%$ of the gamma sensitivity for neutrons at $2 \mathrm{MeV}[6,9]$. This detector was connected to the high-voltage supply unit and the pulse counter, and it was exposed between 10 and 60 min.

\subsubsection{Thermoluminescence dosemeters}

The thermoluminescence dosemeter (TLD), model UD-200S, is manufactured by Panasonic, Ltd., Japan, mainly for the purpose of environmental monitoring. It has high gamma sensitivity and low neutron sensitivity since a phosphor of $\mathrm{CaSO}_{4}(\mathrm{Tm})$ has a small neutron kerma factor and is enclosed in glass material to exclude extraneous recoil protons and other heavy charged particles. The sensitivity for tissue kerma from ${ }^{252} \mathrm{Cf}$ neutrons was estimated as less than $1 \%$ of the gamma sensitivity [10]. The gamma energy response for air kerma is fairly flat within $\pm 30 \%$ from $30 \mathrm{keV}$ to $10 \mathrm{MeV}$ by means of a compensating filter consisting of tin and lead [11]. Three set of TLDs, each composed of a pair of TL elements, were prepared and exposed for $2 \mathrm{~h}$.

\subsubsection{Ionization chamber}

The air-filled ionization-chamber-type gamma-rays ambient dose equivalent rate survey meter, model AE-233V, is manufactured by Applied Engineering, Inc., Japan, for radiation protection purpose. This survey meter has a chamber of $\sim 300 \mathrm{~cm}^{3}$ volume with a 6-mm-thick acrylic resin wall. The gamma energy response for ambient dose equivalent is flat within $\pm 10 \%$ from $30 \mathrm{keV}$ to $1.25 \mathrm{MeV}$, according to the manufacturer's information. In contrast to the abovementioned two dosemeters both constructed of non-hydrogenous materials, the chamber has a hydrogenous wall; thus, the recoil protons produced give the chamber a rather high response to neutrons. Therefore, measured values originating from both gamma-ray and neutron components had to be compensated for. The instrument was surrounded with 5-cm-thick lead blocks, and the net gamma dose rates were determined by subtracting the indicated values obtained with lead shielding from those obtained without this shielding. The indicated values were observed by connecting a digital chart recorder to an output terminal.

\subsection{Calculations}

The air kerma spectra and ambient dose equivalent rates of the primary gamma rays emitted from ${ }^{252} \mathrm{Cf}$ and the secondary gamma rays induced by neutron interaction with the surroundings were calculated by the use of the Monte Carlo code MCNP-4C [12] with the cross-section libraries of MCPLIB02 [13] and ENDF/B-VI [14] for characterizing contaminant photons. 
In the calculation model, all the surrounding structures (the capsule, holder and guide tube) around the ${ }^{252} \mathrm{Cf}$ source and cylindrical moderators were arranged, and the ring detector was set at a distance of $100 \mathrm{~cm}$ from the center of the source to tally the spectral fluence of the photons.

The primary gamma rays with the energy distribution described in ICRU Report 26 [4] and the ${ }^{252} \mathrm{Cf}$ fission neutrons with that given in ISO8529-1 [1] were separately generated from the source region in the mode $p$ and mode $n p$ calculations, respectively. Each calculation result was combined with the emission ratio of the primary gamma rays to the neutrons being 5.7, based on the emission rates of each radiation per unit mass of ${ }^{252} \mathrm{Cf}$ [4]. The air kerma and ambient dose equivalent rate were determined by multiplying the calculated spectral fluence by the fluence-to-air kerma and air kerma-to-ambient dose equivalent conversion coefficients [15] and the neutron emission rate at the time of measurement.

\section{Results and discussion}

\subsection{Calculated gamma-ray spectra}

Figure 1 shows stacked plots of the calculated air kerma spectra along the $y$-axis labeled with the moderator code. The spectrum depicted is a composite of the primary and secondary gamma-ray components, normalized to one neutron emission from the source. The backmost spectrum is the initial gamma-ray spectrum from ${ }^{252} \mathrm{Cf}$. As shown by the trend of stacked spectra, the low-energy part of the primary gamma-ray component was gradually reduced by the presence of thicker and/or heavier moderators, which act as a shield. Meanwhile, the spectra of the moderator code of P100 and F40P60 showed a prominent peak just above $2 \mathrm{MeV}$, which corresponded to $2.2 \mathrm{MeV}$ gamma rays produced by thermal neutron capture by ${ }^{1} \mathrm{H}$ in the acrylic resin via neutron moderation and thermalization. A small bump observed around $7 \mathrm{MeV}$ in the F40P60 spectrum was presumed to be an iron-capture line at $7.63 \mathrm{MeV}$.

Table 2 presents fluence-averaged energies of the primary, secondary, and composite spectra, together with the air kerma ratios between the primary and secondary gamma-ray components. Although the average energies of the primary components remained relatively unchanged by the presence of the moderators, those of the secondary components were highly dependent on the materials. The secondary gamma rays escaping from the acrylic resin moderator had an average energy that monotonically increased by $\mathrm{H}(\mathrm{n}, \gamma)$ with increasing the moderator thickness, while those escaping from the steel moderator were almost of the same energy as the 847 $\mathrm{keV}$ inelastic-scattering gamma rays corresponding to the difference in energy levels between the first excited state and the ground state of ${ }^{56} \mathrm{Fe}$. Despite the presence of such high-energetic secondary gamma rays, fluence-averaged energies of the composite spectra were in the $0.6-0.9 \mathrm{MeV}$ range. Over $70 \%$ of the dose was delivered by gamma rays having energies between 0.1 and $2 \mathrm{MeV}$, where the dosemeter's response does not vary significantly as a function of the gamma-ray energy. The calibration of all three gamma dosemeters was therefore based on ${ }^{137} \mathrm{Cs}$ gamma rays in converting their readings to the dose equivalent.

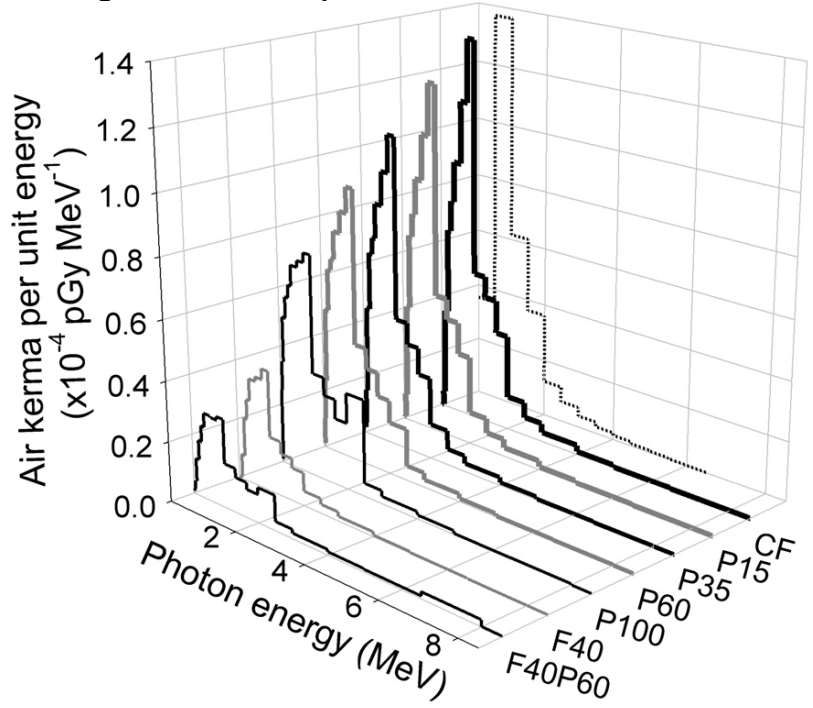

Figure 1. Photon air kerma spectra calculated by the use of MCNP-4C. The backmost dotted line shows the initial primary gamma-rays given in ICRU Report 26 [4].

Table 2. Fluence-averaged energy of the contaminant photons.

\begin{tabular}{|c|c|c|c|c|c|}
\hline \multirow{2}{*}{$\begin{array}{l}\text { Moderator } \\
\text { ID code }\end{array}$} & \multicolumn{3}{|c|}{ Mean energy $(\mathrm{MeV})$} & \multirow{2}{*}{$\begin{array}{c}2^{\text {nd }} / 1^{\text {st }} \\
\text { Dose ratio }\end{array}$} & \multirow{2}{*}{$\begin{array}{c}0.1-2 \mathrm{MeV} \\
\text { Dose fraction }\end{array}$} \\
\hline & $1^{\text {st (a) }}$ & $2^{\text {nd (b) }}$ & Total & & \\
\hline $\mathrm{Cf}$ & 0.70 & 1.21 & 0.70 & 0.021 & $90 \%$ \\
\hline P15 & 0.66 & 1.20 & 0.67 & 0.024 & $90 \%$ \\
\hline P35 & 0.62 & 1.34 & 0.64 & 0.035 & $89 \%$ \\
\hline P60 & 0.58 & 1.49 & 0.61 & 0.076 & $85 \%$ \\
\hline P100 & 0.52 & 1.51 & 0.60 & 0.204 & $77 \%$ \\
\hline F40 & 0.73 & 0.88 & 0.75 & 0.146 & $87 \%$ \\
\hline F40P60 & 0.66 & 1.52 & 0.86 & 0.525 & $69 \%$ \\
\hline
\end{tabular}

(a) Primary gamma ray. (b) Secondary gamma ray.

\subsection{Measurement results}

Table 3 summarizes the values of the ambient photon dose equivalents measured with the three photon dosemeters and calculated with MCNP, as well as the gamma ray to neutron ambient dose equivalent ratios in percentages. The source neutron emission rate was $3.05( \pm 0.7 \%) \times 10^{7} \mathrm{~s}^{-1}$ at the time of measurement (Oct. 26, 2006). The neutron dose equivalent rates in column 2 of Table 3 were based on a previous study [2,3]. The gamma dose equivalent rates observed from the three instruments were in good agreement, and the measurement and calculation results agreed well with each other. The gamma/neutron ratio ranged from 3\% for $\mathrm{F} 40$ to $14 \%$ for $\mathrm{P} 100$ and increased with the thickness of the acrylic resin moderator. This occurred because the gamma dose rate was increased by enhanced emissions of gamma rays by the $\mathrm{H}(\mathrm{n}, \gamma)$ reaction, while the neutron dose rate decreased because of moderation and absorption with increasing thickness. In contrast, the introduction of the steel dramatically reduced the 
Table 3. Ambient dose equivalent rate $H^{*}(10)$ ratios of gamma and neutron $(\gamma / \mathrm{n}$ ratio $)$ at a distance of $1 \mathrm{~m}$.

\begin{tabular}{|c|c|c|c|c|c|c|c|c|c|}
\hline \multirow{2}{*}{$\begin{array}{l}\text { Moderator } \\
\text { ID Code }\end{array}$} & \multirow{2}{*}{$\begin{array}{l}\text { Neutron } \\
H^{*}(10)^{(a)} \\
\left(\mu \mathrm{Sv} \mathrm{h}^{-1}\right)\end{array}$} & \multicolumn{4}{|c|}{ Photon $H^{*}(10)^{(a)}\left(\mu \mathrm{Sv} \mathrm{h}^{-1}\right)$} & \multicolumn{4}{|c|}{$\gamma / \mathrm{n}$ ratio $(\%)$} \\
\hline & & GM-1 & AE-233V & UD-200S & $\begin{array}{l}\text { MCNP } \\
\text { calc. }\end{array}$ & GM-1 & AE-233V & UD-200S & $\begin{array}{l}\text { MCNP } \\
\text { calc. }\end{array}$ \\
\hline $\mathrm{Cf}$ & 370 & $16.3 \pm 0.8^{(\mathrm{b})}$ & $16.6 \pm 0.4$ & $16.3 \pm 0.3$ & 18.5 & $4.4 \pm 0.2^{(\mathrm{b})}$ & $4.5 \pm 0.1$ & $4.4 \pm 0.1$ & 5.0 \\
\hline P15 & 328 & $16.9 \pm 0.8$ & $16.3 \pm 0.4$ & n. m. ${ }^{(c)}$ & 17.5 & $5.2 \pm 0.3$ & $5.0 \pm 0.1$ & - & 5.4 \\
\hline P35 & 254 & $16.2 \pm 0.8$ & $14.8 \pm 0.4$ & $13.9 \pm 0.2$ & 16.4 & $6.4 \pm 0.3$ & $5.8 \pm 0.1$ & $5.5 \pm 0.1$ & 6.5 \\
\hline P60 & 187 & $15.9 \pm 0.8$ & $14.0 \pm 0.3$ & n. m. & 15.6 & $8.5 \pm 0.4$ & $7.5 \pm 0.2$ & - & 8.5 \\
\hline P100 & 110 & $15.2 \pm 0.8$ & $14.5 \pm 0.3$ & $13.9 \pm 0.2$ & 15.2 & $13.9 \pm 0.7$ & $13.2 \pm 0.3$ & $12.7 \pm 0.2$ & 13.9 \\
\hline F40 & 380 & $9.5 \pm 0.6$ & $6.8 \pm 0.4$ & $7.0 \pm 0.2$ & 6.4 & $2.5 \pm 0.2$ & $1.8 \pm 0.1$ & $1.8 \pm 0.0$ & 1.7 \\
\hline F40P60 & 145 & $6.9 \pm 0.6$ & $7.3 \pm 0.3$ & $8.1 \pm 0.1$ & 6.6 & $4.8 \pm 0.4$ & $5.1 \pm 0.2$ & $5.6 \pm 0.1$ & 4.5 \\
\hline
\end{tabular}

(a) Neutron and gamma ambient dose equivalent rates were as of Oct. 26, 2006.

(b) Uncertainties are presented as $\mathrm{k}=1$, derived from statistical uncertainties or standard deviations of readings of dosemeters.

(c) Not measured.

primary gamma rays emitted from the source itself. This table demonstrated good agreement of the dose equivalents measured by the three different instruments and hence the accuracy of the transport calculation. This result also confirmed that the neutron sensitivity of both the GM detector and the TLD to ${ }^{252} \mathrm{Cf}$ fission neutrons can be regarded as nearly zero and that differential measurements by an ionization-chamber-type survey meter with and without a lead shield are a practical photon dosimetry method with a good photon-neutron discrimination capability.

\section{Conclusion}

The authors performed a complementary study to determine the proportion of the contaminating photons present in a moderated neutron field produced by a ${ }^{252} \mathrm{Cf}$ neutron source surrounded with moderators. These photons may affect the readings of survey meters or personal dosemeters.

The photon dose rates were measured with three types of instruments based on different detection principles, and the results were compared with those obtained from Monte Carlo calculations. The contaminant photon dose rates observed from each instrument were in good agreement, and the measurement and calculation results also agreed well. The ambient dose equivalent rate ratio of photon and neutron $(\gamma / \mathrm{n})$ ranged from $3 \%$ to $14 \%$. This information is useful for testing and characterizing a neutron-measuring device with photon sensitivity.

\section{References}

[1] ISO, Reference neutron radiations -Part 1: Characteristics and methods of production, ISO8529-1 (2001).

[2] N. Tsujimura, T. Yoshida and C. Takada, Development of moderated neutron calibration fields simulating workplaces of MOX fuel facilities, Hoken Butsuri (Jpn. J. Health Phys.) 40 (2005), pp. 354-359. [in Japanese]

[3] N. Tsujimura, T. Yoshida, C. Takada, T. Nunomiya and K. Aoyama, Performance test of the electronic personal neutron dosemeter in neutron fields simulating workplaces of MOX fuel fabrication facilities, Proc. 12th Int. Congress of Int. Radiat. Prot. Asso. (IRPA-12), Buenos Aires, Argentina, Sep. 19-24, 2008, (2008). [CD-ROM]

[4] ICRU, Neutron dosimetry for biology and medicine, ICRU Report 26 (1977).

[5] E. B. Wagner and G. S. Hurst, A Geiger-Müeller $\gamma$-ray dosimeter with low neutron sensitivity, Health Phys. 5 (1961), pp. 20-26.

[6] J. C. McDonald, R. V. Griffith, P. A. Plato and J. A. Miklos, Measurement of gamma ray dose from a moderated ${ }^{252} \mathrm{Cf}$ source, Radiat. Prot. Dosim. 9 (1984), pp.113-118.

[7] C. J. Mckay, P. Burgess, N. P. Hawkes, M. Kelly, G. C. Taylor and D. J. Thomas, Photon doses in NPL standard radionuclide neutron fields, NPL Report IR 12 (2009).

[8] N. Tsujimura and T. Yoshida, Evaluation of neutron response of criticality accident alarm system detector, Prog. Nucl. Sci. Technol. (this issue)

[9] S. Guldbakke, R. Jahr, H. Lesiecki and H. Schölermann, Neutron response of Geiger-Müller photon dosemeters for neutron energies between $100 \mathrm{keV}$ and $19 \mathrm{MeV}$, Health Phys. 39 (1980), pp. 963-969.

[10] T. Irifune and Y. Onai, Sensitivity of various $\gamma$-ray detectors to ${ }^{252} \mathrm{Cf}$ neutrons in free air, Radioisotopes 25 (1976), pp.552-555.

[11] T. Yamashita, N. Noda, H. Onishi and S. Kitamura, Calcium sulfate activated by thulium or dysprosium for thermoluminescence dosimetry, Health Phys. 21 (1971), pp.295-300.

[12] J. F. Briesmeister, ed., MCNP - A general Monte Carlo $\mathrm{N}$-particle transport code, version $4 \mathrm{C}$, LA-13709-M, Los Alamos National Laboratory, (2000).

[13] H.G. Hughes, Information on the photon library MCPLIB02, LANL X-6: HGH-93-77, (1996).

[14] J. S. Hendricks, S. C. Frankle and J. D. Court, $E N D F / B-V I$ data for MCNP, LA-12891, Los Alamos National Laboratory (1994).

[15] ICRU, Conversion coefficients for use in radiological protection against external radiation, ICRU Report 57 (1998). 